\title{
How Does Evolutionary Computation Fit Into IT Postgraduate Teaching
}

\author{
Xin Yao \\ School of Computer Science \\ The University of Birmingham \\ Edgbaston, Birmingham B15 2TT \\ UK \\ xin@cs.bham.ac.uk
}

\begin{abstract}
Evolutionary computation courses have been offered by a wide range of departments/schools to students with many different backgrounds. This paper describes three postgraduate courses with significant evolutionary computation components offered by the University College of the University of New South Wales at the Australian Defence Force Academy. The courses have been offered as part of the Master of Science in Information Technology programme and Master of Science in Operations Research and Statistics programme. This paper also summarises the result of a recent survey on evolutionary computation teaching conducted over the Internet.
\end{abstract}

\section{Introduction}

Although the history of evolutionary computation (EC) can be traced back to 1950's [1], EC courses have only been offered in universities in the last decade or so. There has been a dramatic increase in the number of universities offering EC or EC-related courses in recent years [2]. However, there have been no standard teaching methods or textbooks for EC courses. Different lecturers tend to use quite different teaching methods, textbooks, reading materials and assessment methods according to the background of students. There is an urgent for lecturers of EC courses to exchange their ideas, share their experience and discuss their opinions on how to teach an EC course.

The University College of the University of New South Wales (UNSW) is located within the Australian Defence Force Academy in Canberra, Australia. It has offered three EC-related courses to postgraduate students in the last few years. These courses have been offered as part of the Master of Science in Information Technology programme and Master of Science in Operations Research and Statistics programme. This paper will describe these three courses in the following sections and finish with a brief summary of a recent survey on evolutionary computation teaching conducted over the Internet [2].

\section{Postgraduate Course on Evolutionary Compu- tation}

The course, entitled "Evolutionary Computation," was offered in the second semester in 1995. The students were enrolled in either the Master of Science in Information Technology programme or Master of Engineering Science programme. The computing and programming backgrounds of these students varied greatly. Some, being programmers in a government agency, have extensive programming experience, while others have very limited computing skills. Some never used Unix. This mix of student backgrounds was a big challenge to the lecturer (author) in designing and delivering the course. It was decided that the assumption of first-year computer science and first-year mathematics would apply, which turned a few students away.

The course consisted of two hours lecture and one hour lab session each week. The topics covered include:

- Theoretical analysis of GAs, including hyperplane sampling, implicit parallelism, search space as hypercubes, schema theorem, premature convergence, etc.

- Variants of the canonical genetic algorithm, including various crossover and mutation operators, selection schemes, generation gap, adaptive population size, adaptive control parameters, etc.

- Fitness scaling, niche, fitness sharing and coevolution, inclduing multiple-peak optimisation, speciation, explicit and implicit fitness sharing, etc.

- Evolutionary programming and simulated annealing, including function optimisation, selfadaptation, fast simulated annealing, very fast simulated annealing and comparative studies.

- Genetic algorithms for game strategy learning, including the 2-player and $N$-player $(N>2)$ iterated prisoner's dilemma games using GAs and EP.

- Evolution strategies, including $(\lambda+\mu)$-ES and $(\lambda, \mu)$-ES, self-adaptation, function optimisation, etc.

- Evolutionary artificial neural networks, including the evolution of weights, architectures, and learning rules.

- Learning classifier systems, including credit assignment, default hierarchy, message passing, etc.

- Population-based machine learning and evolutionary robotics.

The course was largely project-based. $40 \%$ of the as- 
sessment was awarded according to a project. The students were free to choose their own projects and then consulted with the lecturer on their suitability. Most students chose a project which applied an evolutionary algorithm to their problem at work, e.g., aircraft landing scheduling at the Sydney airport, automated cryptanalysis of polyalphabetic substitution ciphers, 3-d terrain traversal, ADFA lecture room assignment, etc. The work produced by some students were very impressive and later published in conference proceedings. The course also included a reading and presentation of a recent technical paper, and two other assignments as the remaining part of the assessment.

In the lab sessions, students started with a given software package. We used Bäck's GENEsYs for doing simple exercises and experiments. For the project work, most students wrote their own software, which were rather demanding for people without a solid background in computer science and programming.

\section{Postgraduate Course on Machine Learning}

This course was first offered in Semester 1 of 1995. It was designed to introduce a number of different machine learning techniques to students with little artificial intelligence background. The emphasis was put on the practical rather than theoretical side of the techniques.

The three major components of the course are propositional learning (mainly decision trees, given by $\mathrm{Dr}$ Robert Pearson), relational learning (mainly inductive logic programming, given by $\mathrm{Dr}$ Bob McKay), and stochastic learning (neural and evolutionary learning, given by the author). In the evolutionary learning part, there was no coverage of optimisation. The emphasis was put on how evolution could be used in supervised, reinforcement and unsupervised learning, how the evolutionary approach differed from other approaches, and how to combine different approaches. It was quite interesting to discuss the similarities and differences between the evolutionary approach and other approaches in machine learning. The generalisation issue was used as the primary theme throughout the whole course.

\section{Postgraduate Course on Modern Heuristic Techniques}

This course was offered in 1998 as part of the Master of Science in Operations Research and Statistics programme. It was based on the book edited by Reeves [3]. The course has a two hour lecture and a one hour lab session each week. The following is taken from our handbook:

This unit covers a number of new heuristic techniques in operations research, especially in optimisation and scheduling. These new techniques have been proposed to deal with nonlinear and complex problems where traditional programming methods failed. The topics covered in this unit include: the theory and applications of simulated annealing, fast and very fast simulated annealing, genetic algorithms, evolutionary programming, evolution strategies, tabu search, Hopfield-type artificial neural networks, and mean-field annealing.

The assessment of the course was based on a project, student presentation and an assignment. Both the project and the assignment required programming and developing a software package, which made this course rather different from most other courses in the Operations Research and Statistics programme. However, the feedback from students was very good because they learned some new techniques which can be applied to solve real-world problems. For example, one student did a project on optimal mixing of different feeds for horses, a problem which had always been tackled through trialand-error.

\section{Evolutionary Algorithms as Generate-and- Test}

There are a couple of things we did in our courses that we find useful. One is not to introduce evolutionary algorithms (EAs) from the biological point of view, but from the search point of view in order to link EAs with other well-known algorithms in computer science. The other is to put evolutionary learning firmly in the general framework of machine learning and use generalisation as the central theme in evolutionary learning. This section will outline our idea of treating EAs as a special class of generate-and-test search algorithm when we first introduce EAs. The next section will describe how the emphasis on generalisation can lead to better understanding of the potential strength and weakness of evolutionary learning.

Although EAs are often introduced from the point of view of survival of the fittest and from the analogy to natural evolution, they can also be understood through the framework of the generate-and-test search. The advantage of introducing EAs as a type of generate-andtest search algorithms is that the relationships between EAs and other search algorithms, such as simulated annealing, tabu search, hill-climbing, etc., can be made clearer and thus easier to explore. Under the framework of generate-and-test search, different search algorithms investigated in artificial intelligence, operations research, computer science, and evolutionary computation can be unified together. Cross-interdisciplinary studies are expected to generate more insights into the search problem in general. A general framework of generate-and-test 
search is shown by Figure 1.

1. Generate the initial solution at random and denote it as the current solution;

2. Generate the next solution from the current one by perturbation;

3. Test whether the newly generated solution is acceptable;

(a) Accepted it as the current solution if yes;

(b) Keep the current solution unchanged otherwise.

4. Goto Step 2 if the current solution is not satisfactory, stop otherwise.

Figure 1: A General Framework of Generate-and-Test.

It is quite clear that various hill-climbing algorithms can be described by Figure 1 with different strategies for perturbation. They all require the new solution to be no worse than the current one to be acceptable. SA does not have such a requirement. It regards a worse solution to be acceptable with certain probability. The difference among classical SA [4], fast SA [5], very fast $\mathrm{SA}[6]$, and a new SA [7] is mainly due to the difference in their perturbations, i.e., methods of generating the next solution.

EAs can be regarded as a population-based version of generate-and-test search. They use search operators like crossover and mutation to perturb the current solutions, and use selection to decide whether a solution should be accepted. From this point of view, it is clear that we do not have to limit ourselves to crossover and mutation. In theory, we can use any search operators as long as they perform well on the given representation of the problem they are dealing with. This is also true for selection. In practice, a good way to tailor the generate-and-test search to the problem we are interested in is to incorporate problem specific heuristic knowledge into search operators and selection schemes[8].

The generate-and-test view of EAs has been shown to be very effective in getting the basic ideas of EAs across to students. It is easier for students to understand EAs when they can relate EAs to other problem solving algorithms they know. As a result, students are more likely to design and develop their own search operators in their project work. Hybrid algorithms become something very natural and common because everything comes down to the issue of how to generate better offspring with higher probabilities and how to select (i.e., test) individuals with higher probabilities of leading towards the global optimum.

The generate-and-test view of EAs also reveals the statistical sampling nature of EAs. It tells students that an EA needs sufficient sampling in order to obtain statistically more accurate information to guide further search. Running EAs for only a few generations and getting a very good result usually imply that there exists a better algorithm (either a deterministic or random one) for solving the problem.

\section{Exploit Population Information}

Learning is often formulated as an optimisation problem in the machine learning field. For example, backpropagation (BP) is often used to train feed-forward ANNs [9]. This training process is also called the learning process of ANNs. BP is known as one of the most widely used learning algorithms. However, BP is in essence a gradient-based optimisation algorithm which is used to minimise an error function (often a mean square error) of ANNs. The so-called learning problem here is a typical optimisation problem in numerical analysis. Many improvements on the ANN learning algorithm are actually improvements over optimisation algorithms [10], such as conjugate gradient methods $[11,12]$.

Learning is different from optimisation because we want the learned system to have best generalisation, which is different from minimising an error function. The ANN with the minimum error does not necessarily mean that it has best generalisation unless there is an equivalence between generalisation and the error function. Unfortunately, measuring generalisation exactly and accurately is almost impossible in practice [13], although there are many theories and criteria on generalisation, such as the minimum description length (MDL) [14], Akaike information criteria (AIC) [15], and minimum message length (MML) [16]. In practice, these criteria are often used to define better error functions in the hope that minimising the functions will maximise generalisation. While better error functions often lead to better generalisation of learned systems, there is no guarantee. Regardless of the error functions used, BP or other more advanced learning algorithms are still used as optimisation algorithms. They just optimise different error functions. The nature of the problem is unchanged.

Similar situations occur with other machine learning methods, where an "error" function has to be defined. A "learning" algorithm then tries to minimise the function. However, no error functions can guarantee that they correspond to the true generalisation [13]. This is a problem faced by most inductive learning methods. There is no way in practice one can get around this except for using a good empirical function which might not correspond to the true generalisation. Hence, formulating learning as optimisation in this situation is justified.

Evolutionary learning is a population-based learning method. Most people use an evolutionary algorithm to maximise a fitness function or minimise an error func- 
tion, especially in supervised learning, and thus face the same problem as that described above. EAs were used in essence as optimisation algorithms. While little can be done for traditional non-population-based learning, there are opportunities for improving population-based learning, e.g., evolutionary learning.

Since the maximum fitness is not equivalent to best generalisation in evolutionary learning, the best individual with the maximum fitness in a population may not be the best one in terms of generalisation. Other individuals in the population may contain some useful information that will help to improve generalisation of learned systems. It is thus beneficial to make use of the whole population rather than any single individual. A population always contains at least as much information as any single individual. This population point of view has led to various evolutionary approaches to designing machine learning systems with many subsystems, such as neural network ensembles and committees [17]. These approaches are quite different from learning classifier systems in which each individual is a single rule.

The discussion on the difference between optimisation and learning in terms of generalisation has helped students to link evolutionary learning with other types of learning and see the potential advantages and disadvantages of evolutionary learning better.

\section{EC Courses in the World - A Brief Look}

Riccardo Poli and I conducted a survey on various EC-related courses offered by universities between last November and this January. The survey form was posted to various news groups and mailing lists through Internet. The original call-for-information is attached at the end of this paper.

A total of 37 email responses were received by the middle of last December, which included 38 different ECrelated courses (not including the three courses described in previous sections of this paper). Although this is not a complete list of EC courses offered in the world due to the short time frame we had, they certainly represent a significant portion of all the EC-related courses. According to the topics covered by different courses, we can group courses into four broad categories: (1) those almost entirely on EC techniques, (2) those on optimisation techniques, including EAs, neural networks (NNs), simulated annealing (SA), tabu search, etc.; (3) those on machine learning techniques, including EAs, NNs, decision trees (DT), inductive logic programming (ILP), cellular automata (CA), etc.; and (4) other courses with a significant EC component. Table 1 shows the number of courses in each category.

The number of students in each course varied greatly from 4 to 40 . There was a research-based course which only had one or two students each year. The back- ground of students was mostly in computer science although students from other backgrounds also took EC courses. They included engineering, economics, water resources management, hydrology, information systems, industrial engineering and management, architecture, biochemistry, business, physics, mathematics, applied mathematics, medical information sciences, etc.

Ten of the 38 courses were offered by US universities. Six were offered by UK universities. The rest were from Germany, Poland, Denmark, Australia, Austria, Spain, Italy, Holland, Mexico, France, India and Brazil.

The most popular textbook or recommended reading appears to be Michalewicz's book [18], followed by Goldberg's book [19]. Other books used by various courses include (not an exhaustive list): Mitchell's [20], Fogel's $[21,1]$, Schwefel's [22] and Koza's [23]. However, most lecturers used their own notes or handouts. Less than half of the courses used the same textbook or recommended reading material.

\section{Conclusions}

We have found that an EC course can be incorporated into an postgraduate information technology programme quite nicely since EC techniques are closely related to many existing problem solving techniques.

\section{Acknowledgements}

The three courses described in this paper were offered while the author was with the School of Computer Science, University College, The University of New South Wales, Australian Defence Force Academy, Canberra, ACT 2600, Australia.

\section{Bibliography}

[1] D. B. Fogel, Evolutionary Computation: The Fossil Record. Piscataway, NJ, USA: IEEE Press, 1998.

[2] R. Poli and X. Yao (ed.), "Evolutionary computation teaching survey," tech. rep., http://www.cs.bham.ac.uk/ rmp/eebic/ec_teaching/, 1999.

[3] C. R. Reeves, ed., Modern Heuristic Techniques for Combinatorial Problems. Oxford OX2 0EL: Blackwell Scientific, 1993.

[4] S. Kirkpatrick, C. D. Gelatt, and M. P. Vecchi, "Optimization by simulated annealing," Science, vol. 220, pp. 671-680, 1983.

[5] H. H. Szu and R. L. Hartley, "Fast simulated annealing," Physics Letters A, vol. 122, pp. 157-162, 1987. 


\begin{tabular}{|c|c|c|c|}
\hline $\begin{array}{c}\text { Almost entirely } \\
\text { on EC }\end{array}$ & $\begin{array}{c}\text { Optimisation Techniques } \\
\text { EC+NN+SA+Tabu+... }\end{array}$ & $\begin{array}{c}\text { Learning Techniques } \\
\text { EC+NN+CA+DT+... }\end{array}$ & Others \\
\hline 24 & $\mathbf{3}$ & 9 & 2 \\
\hline
\end{tabular}

Table 1: Different courses according to the topics covered.

[6] L. Ingber, "Very fast simulated re-annealing," Mathl. Comput. Modelling, vol. 12, no. 8, pp. 967973, 1989.

[7] X. Yao, "A new simulated annealing algorithm," Int. J. of Computer Math., vol. 56, pp. 161-168, 1995.

[8] J. J. Grefenstette, "Incorporating problem specific knowledge into genetic algorithms," in Genetic Algorithms and Simulated Annealing (L. Davis, ed.), ch. 4, pp. 42-60, San Mateo, CA: Morgan Kaufmann, 1987.

[9] D. E. Rumelhart, G. E. Hinton, and R. J. Williams, "Learning internal representations by error propagation," in Parallel Distributed Processing: Explorations in the Microstructures of Cognition, Vol. I (D. E. Rumelhart and J. L. McClelland, eds.), pp. 318-362, MIT Press, Cambridge, MA, 1986.

[10] D. R. Hush and B. G. Horne, "Progress in supervised neural networks," IEEE Signal Processing Magazine, vol. 10, no. 1, pp. 8-39, January 1993.

[11] E. M. Johansson, F. U. Dowla, and D. M. Goodman, "Backpropagation learning for multi-layer feed-forward neural networks using the conjugate gradient method," Int'l J. of Neural Systems, vol. 2, no. 4, pp. 291-301, 1991.

[12] M. F. Møller, "A scaled conjugate gradient algorithm for fast supervised learning," Neural Networks, vol. 6, pp. 525-533, 1993.

[13] D. H. Wolpert, "A mathematical theory of generalization," Complex Systems, vol. 4, pp. 151-249, 1990.

[14] J. Rissanen, "Modeling by shortest data description," Automatica, vol. 14, pp. 465-471, 1978.

[15] H. Akaike, "A new look at the statistical model identification," IEEE Trans. Appl. Comp., vol. AC19, pp. 716-723, 1974.

[16] C. S. Wallace and J. D. Patrick, "Coding decision trees," Tech. Rep. 91/153, Dept. of Computer Science, Monash University, Clayton, Victoria 3168, Australia, August 1991.

[17] X. Yao and Y. Liu, "Making use of population information in evolutionary artificial neural networks,"
IEEE Trans. on Systems, Man, and Cybernetics, Part B: Cybernetics, vol. 28, no. 3, pp. 417-425, 1998.

[18] Z. Michalewicz, Genetic Algorithms + Data Structures $=$ Evolution Programs (3rd edition). Berlin, Germany: Springer-Verlag, 1996.

[19] D. E. Goldberg, Genetic Algorithms in Search, Optimization, and Machine Learning. Reading, MA: Addison-Wesley, 1989.

[20] M. Mitchell, An Introduction to Genetic Algorithms. Cambridge, MA, USA: The MIT Press, 1996.

[21] D. B. Fogel, Evolutionary Computation: Towards a New Philosophy of Machine Intelligence. New York, NY: IEEE Press, 1995.

[22] H.-P. Schwefel, Evolution and Optimum Seeking. New York: John Wiley \& Sons, 1995.

[23] J. R. Koza, Genetic Programming. Cambridge, Mass.: The MIT Press, 1992. 